\title{
Improving the approach to non-diagnostic aspirates: learning from each other
}

\author{
Erik K. Alexander ${ }^{1}$
}

Received: 16 March 2015/Accepted: 23 March 2015/Published online: 3 April 2015

(C) Springer Science+Business Media New York 2015

The primary purpose of thyroid nodule fine needle aspiration (FNA) is to detect thyroid cancer. This minimally invasive procedure has proven highly safe and effective. Unfortunately, a small proportion of aspirates will prove cytologically non-diagnostic, demonstrating insufficient follicular cells to allow an adequate and interpretable sample. Non-diagnostic aspirates lead to uncertainty and anxiety for both the patient and physician. A benign process cannot be assumed [1]. Clinical guidelines generally suggest repeat aspiration to be performed [2], though variation in clinical practice is known to exist. This is accurately detailed in the study by Brito et al. who present survey data describing the clinical practice patterns of 694 endocrinologists. Most encouraging, physicians generally report a non-diagnostic rate less than $10 \%$, and recommend repeat FNA of such nodules 1-3 months thereafter [3]. High volume activity ( $>100$ FNA's per year) predicts the lowest risk of non-diagnostic results. Yet, these data also demonstrate variability in practice, and provide insight for improvement.

It is well documented that the greatest risk factor predicting a non-diagnostic aspirate is the nodule's cystic content [4]. Cyst fluid often contains necrotic debris or hemorrhagic material, but rarely any viable cellular material. Such cystic nodules are almost always benign ( $>98 \%$ of cases), and will often regress without intervention. Thus, the approach to a non-diagnostic cystic nodule should be conservative, rarely requiring surgical removal. Many cystic nodules can be effectively drained,

Erik K. Alexander

ekalexander@partners.org

1 Brigham \& Women's Hospital, Harvard Medical School, Boston, USA while others describe the utility of sclerosis via sterile ethanol injection [5].

Importantly, Brito's investigation is specific to solid thyroid nodules, where non-diagnostic aspirates are less common. Nonetheless, coarse or rim calcifications as well as proximity to important anatomic structures (such as the carotid artery or trachea) can limit the ability of the practitioner to obtain adequate tissue. In some cases, no cause can be identified. In these scenarios, repeat evaluation of the solid nodule is indicated, as others have described a persistent risk of malignancy in such circumstances [1]. Brito et al. nicely depict the options available to the clinician as they seek diagnostic material. Most often, repeat aspiration is performed, often adding additional needle sticks. Separately, ensuring ultrasound guidance of the needle to the solid, cellular area of the nodule is important. Other practitioners arrange for onsite cytologic analysis, which can provide real-time identification of adequate tissue. All approaches are valid, and often occur in combination. As is noted with many procedures, high volume leads to improved results. Thus, it is encouraging to also see that some practitioners refer patients with a non-diagnostic aspirate to a colleague for assistance.

Two findings in the survey clarify areas for educational development. Most physicians surveyed recommended follow up and repeat assessment in 1-3 months. While reasonable from a practice management standpoint and with regards to patient concern, longer time frames for follow up are likely reasonable. Most thyroid cancers are low risk, indolent malignancies unlikely to rapidly change. Unless sonographically high risk (or symptoms suggest the rare possibility of distant metastases), a longer time interval should be viewed as equally acceptable. In support, prior investigations have confirmed with utility and safety 
of repeat aspirations of cytologically 'atypical' (AUS/ FLUS) nodules at 6 months [6]. Thus, such a strategy for sonographically low-risk, non-diagnostic aspirates seems equally justified.

Finally, it is notable that $56 \%$ of respondents favored research toward the development of a molecular diagnostic test usable in this setting. While the power of molecular diagnostics should not be underestimated, it is critical to note that molecular analysis requires adequate tissue sampling not too different from cytologic assessment. Molecular tests can similarly return with a nondiagnostic result. Furthermore, no molecular diagnostic strategy is likely to supplant the primacy of FNA cytology for initial nodule evaluation given its relatively low cost, rapid interpretation, and impressive accuracy when cytologically benign [7].

In summary, Brito et al. provide important insight into the clinical strategies currently used to evaluate solid, yet cytologically non-diagnostic thyroid nodules. It is likely that such strategies would (and should) differ for cystic or partially cystic nodules. These data demonstrate the successful implementation of evidence-based clinical guidelines into daily practice, depicting validated strategies used by physicians to improve diagnostic yield. Sonographic nodule assessment and needle targeting, additional needle aspirates, and onsite cytology assessment are all validated approaches. Yet, the diversity of findings, and the respondents desires for relatively rapid repeat assessment suggest continuing medical education and development can benefit this community of physician. By doing so, the health of the many individuals who suffer from this common disease will be improved.
Disclosure Dr. Alexander has served as a consultant for NPS Pharma, and Veracyte, Inc.

\section{References}

1. M.W. Yeh, O. Demircan, P. Ituarte, O.H. Clark, False-negative fine-needle aspiration cytology results delay treatment and adversely affect outcome in patients with thyroid carcinoma. Thyroid 14, 207-215 (2004)

2. D.S. Cooper, G.M. Doherty, B.R. Haugen, R.T. Kloos, S.L. Lee, S.J. Mandel, E.L. Mazzaferri, B. McIver, F. Pacini, M. Schlumberger, S.I. Sherman, D.L. Steward, R.M. Tuttle, Revised American Thyroid Association management guidelines for patients with thyroid nodules and differentiated thyroid cancer. Thyroid 11, 1167-1214 (2009)

3. J.P. Brito, M.R. Castro, D.S. Dean, V. Fatourechi, M. Stan, Survey of current approaches to non-diagnostic fine-needle aspiration from solid thyroid nodules. Endocrine (2015). doi:10.1007/ s12020-015-0539-4

4. E.K. Alexander, J.P. Heering, C.B. Benson, M.C. Frates, P.M. Doubilet, E.S. Cibas, E. Marqusee, Assessment of non-diagnostic ultrasound-guided fine needle aspiration of thyroid nodules. J. Clin. Endocrinol. Metab. 87, 4924-4927 (2002)

5. W. Zieleźnik, A. Kawczyk-Krupka, M.P. Barlik, W. Cebula, A. Sieroń, Modified percutaneous ethanol injection in the treatment of viscous cystic thyroid nodules. Thyroid. 15, 683-686 (2005)

6. L. Yassa, E.S. Cibas, C.B. Benson, M.C. Frates, P.M. Doubilet, A.A. Gawande, F.D. Moore Jr, B.W. Kim, V. Nosé, E. Marqusee, P.R. Larsen, E.K. Alexander, Long-term assessment of a multidisciplinary approach to thyroid nodule diagnostic evaluation. Cancer 111, 508-516 (2007)

7. Z.W. Baloch, V.A. LiVolsi, S.L. Asa, J. Rosai, M.J. Merino, G. Randolph, P. Vielh, R.M. DeMay, M.K. Sidawy, W.J. Frable, Diagnostic terminology and morphologic criteria for cytologic diagnosis of thyroid lesion: a synopsis of the National Cancer Institute Thyroid Fine-Needle Aspiration State of the Science Conference. Diagn. Cytopathol. 36, 425-437 (2008) 